\title{
Some additions to the Scale insect fauna (Hemiptera: Coccoidea) of Crete with a check list of the species known from the island
}

\begin{abstract}
A list of the scale insects (Homoptera: Coccoidea) recorded by the authors for the Greek island of Crete is reported. This includes twenty-seven species new to the island The most interesting records are Kermes palestiniensis Balachowsky (Kermesidae), only recorded previously from Israel, and Getulaspis bupleuri (Marchal) (Diaspididae), only known previously from North Africa and the Middle East. With the present additions, the number of scale insect species recorded on Crete has reached 82. A revised check list of the scales presently known from the island is also provided.

Riassunto - Nuovi reperti per la fauna di Homoptera Coccoidea di Creta e check list delle specie note nell'isola

Viene riportato l'elenco di cocciniglie (Hemiptera, Coccoidea) raccolte dagli autori, in periodi diversi, nell'isola di Creta (Grecia). Di queste, 27 sono le specie non ancora note per l'isola. Di particolare interesse biogeografico è il reperimento di Kermes palestiniensis, Balachowsky (Kermesidae), noto finora solo per Israele e di Getulaspis bupleuri (Marchal) (Diaspididae), nota per Nordafrica e Medio Oriente. Viene presentata una check list delle cocciniglie finora note per l'isola.
\end{abstract}

Key words: Greece, Kermes palestiniensis, Getulaspis bupleuri

This paper deals with the scale insect species collected by the authors on Crete at different periods. These records stimulated a deeper study on the scale insect fauna of this Mediterranean island, as it was apparent that the present knowledge on this subject was poor and largely incomplete. According to ScaleNet (Ben-Dov et al., 2010), there are only 43 scale insect species recorded from Crete. Recently Jansen et al. (2011) added six species to this list, raising to 49 the total number of species known to occur on the island. This number is much lower than expected. Even very common, widely distributed species in the Mediterranean basin, and significant agricultural and forestry pest species had not been recorded there, although they are generally listed for the Greek fauna.

Several authors (Podsiadlo, 1983; Kozár et al., 1991; Kozár \& Nagy, 1998) have provided valuable contributions to the knowledge of Greek scale insects, but many 
records from Crete were either overlooked or had been included in the Greek mainland fauna (Ben-Dov et al., 2010). On the other hand, Milonas et al. (2008) list 168 scale insect species for the whole of Greece. This list includes also species only known to occur in Crete.

Argyriou (1984), in her paper dealing with scales of agricultural plants in Greece, only mentions explicitly Lichtensia viburni Signoret, Filippia follicularis Targioni Tozzetti, Marchallina hellenica Gennadius and Pollinia pollini (A. Costa) as being present in Crete. Other common agricultural scale pests are reported as being "widely distributed all over the country" (i.e. Sphaerolecanium prunastri (Fonscolombe) and Planococcus ficus (Signoret)), possibly inferring that they are present also in Crete.

From a biogeographic point of view, the flora and fauna of the larger Mediterranean islands (Corsica, Sardinia, Sicily, Crete, Cyprus) are kept in separated lists from their mainland country of pertinence as they often host endemic species.

Due to the uncertainty of the occurrence of some species in Crete, each bibliographic source recorded in ScaleNet, as being present in Crete, has been checked by reading the original paper. This has led to the discovery of several trivial mistakes, so that species clearly referred in the original papers as being present on the Greek mainland only, have been listed for Crete, (i.e. Eumyrmococcus corinthiacus Williams (Williams, 1993), Eulecanium ciliatum Douglas and Scythia festuceti (Šulc) (Kozár et al., 1991)). In addition, species recorded from Crete only have been listed for mainland Greece (i.e Rhizococcus agropyri Borchsenius), whilst other species are not known from either mainland Greece or Crete (i.e. Atrococcus arakelianae (Ter-Grigorian) (Kozár \& Nagy, 1998)).

The present paper lists the species collected by the authors in Crete, along with the localities, collecting dates and host plants. Species newly recorded from the island are marked with an asterisk. A provisional check-list of the scale insects now known from Crete, along with bibliographic sources, is given in Table 1. Erroneous and uncertain previous records have been omitted.

Among the collected species, Diaspidiotus lenticularis and Lepidosaphes flava were infected by Septobasidium sp., a fungus often associated with armoured scale insects, and which changes the scale morphology (Couch, 1938).

\section{Fam. Ortheziidae}

*Orthezia urticae (Linnaeus): Knossos, 25.ix.2009, undetermined plant (F. Kozár).

\section{Fam. Monophlebidae}

Gueriniella serratulae (Fabricius): Vai, 10.iv.2010, Genista acanthoclada (G. Pellizzari). Previously recorded in Crete (Stalis) by Podsiadlo (1983).

Icerya purchasi Maskell: Iraklion, 21-26.ix.2009, Citrus (F. Kozár); Chania, 8.iv.2010, Pittosporum tobira (G. Pellizzari).

\section{Fam. Marchalinidae}

Marchalina hellenica Gennadius: High infestations were observed at Agios Ioannis, 7.iv.2010, Pinus brutia (G. Seljak); Anopoli, 7.iv.2010, Cupressus sp., Pinus halepensis 
Table 1 - Species recorded in Crete and validation source

Fam. Ortheziidae

\begin{tabular}{|l|l|}
\hline Species & Validation source \\
\hline Orthezia urticae (Linnaeus) & Present paper \\
\hline
\end{tabular}

Fam. Monophlebidae

\begin{tabular}{|l|l|}
\hline Species & Validation source \\
\hline Gueriniella serratulae (Fabricius) & Podsiadlo, 1983; present paper \\
\hline Icerya purchasi Maskell & Ayoutantis, 1940; Podsiadlo, 1983 \\
\hline
\end{tabular}

Fam. Marchalinidae

\begin{tabular}{|l|l|}
\hline Species & Validation source \\
\hline Marchalina hellenica Gennadius & $\begin{array}{l}\text { Argyriou, 1984; Hodgson \& Gounari, } \\
2006\end{array}$ \\
\hline
\end{tabular}

Family Matsucoccidae

\begin{tabular}{|l|l|}
\hline Species & Validation source \\
\hline Matsucoccus josephi Bodenheimer \& Harpaz & Mendel, 1998 \\
\hline
\end{tabular}

Fam. Pseudococcidae

\begin{tabular}{|c|c|}
\hline Species & Validation source \\
\hline Atrococcus arakelianae (Ter-Grigorian) & Kozár \& Nagy, 1998 \\
\hline Coccidohystrix sp.n.? & Present paper \\
\hline Chorizococcus rostellum Lobdell & Present paper \\
\hline Heliococcus bohemicusŠulc & Jansen et al., 2010 \\
\hline Heterococcus nudus Green & Present paper \\
\hline Peliococcus kimmericus (Kiritshenko) & Kozár et al., 1991 \\
\hline Phenacoccus bicerarius Borchsenius & Kozár et al., 1991 \\
\hline Phenacoccus madeirensisGreen & Jansen et al., 2010 \\
\hline Planococcus citri(Risso) & Ayoutantis, 1940 \\
\hline Planococcus ficus (Signoret) & Argyriou, 1984 , \\
\hline Planococcus vovae(Nasonov) & $\begin{array}{l}\text { Williams \& Moghaddam, 2000; } \\
\text { present paper }\end{array}$ \\
\hline Pseudococcus longispinus(Targioni Tozzetti) & present paper \\
\hline Spilococcus halli(McKenzie \& Williams) & Present paper \\
\hline Trionymus aberrans Goux & Kozár et al., 1991 \\
\hline Trionymus multivorus & Present paper \\
\hline
\end{tabular}


Fam. Eriococcidae

\begin{tabular}{|l|l|}
\hline Species & Validation source \\
\hline Acanthococcus sp. n., & Present paper \\
\hline Acanthococcus desertus (Matesova) & Present paper \\
\hline Acanthococcus munroi Boratynski & Kozár et al., 1991 \\
\hline Rhizococcus agropyri Borchsenius & Kozár et al., 1991 \\
\hline Rhizococcus cynodontis Kiritchenko & Kozár et al., 1991 \\
\hline
\end{tabular}

Family Kermesidae

\begin{tabular}{|l|l|}
\hline Species & Validation source \\
\hline Kermes palestiniensis Balachowsky & present paper \\
\hline Kermes vermilio Planchon & $\begin{array}{l}\text { Lindinger, 1912; Hoy, 1963; Present } \\
\text { paper }\end{array}$ \\
\hline
\end{tabular}

Fam. Cerococcidae

\begin{tabular}{|l|l|}
\hline Species & Validation source \\
\hline Cerococcus cistarum Balachowsky & Kozár \& Nagy, 1998 \\
\hline
\end{tabular}

Fam. Coccidae

\begin{tabular}{|l|l|}
\hline Species & Validation source \\
\hline Ceroplastes floridensis Comstock & Present paper \\
\hline Ceroplastes rusci (Linnaeus) & $\begin{array}{l}\text { Ayoutantis, 1940; Podsiadlo, 1983; } \\
\text { present paper }\end{array}$ \\
\hline Ceroplastes sinensis Del Guercio & Present paper \\
\hline Coccus hesperidum Linnaeus & Ayoutantis, 1940 Podsiadlo, 1983 \\
\hline Filippia follicularis (Targioni Tozzetti) & Argyriou, 1984 \\
\hline Lecanopsis formicarum (Newstead) & Present paper \\
\hline Lichtensia viburni Signoret & Argyriou, 1984 \\
\hline Parthenolecanium corni (Bouché) & Kozár et al.,1991 \\
\hline Poaspis intermedia (Goux) & Kozár \& Nagy, 1998 \\
\hline Protopulvinaria pyriformis (Cockerell) & Jansen et al., 2010; present paper \\
\hline Pulvinariella mesembryanthemi (Vallot) & Kozàr et al.,1991 \\
\hline Saissetia coffeae (Walker) & Podsiadlo, 1983 \\
\hline Saissetia oleae (Olivier) & $\begin{array}{l}\text { Ayoutantis, 1940; Argyriou \& } \\
\text { Michelakis, 1975 }\end{array}$ \\
\hline Sphaerolecanium prunastri (Boyer de Fonscolombe) & $\begin{array}{l}\text { Argyriou \& Paloukis, 1976; } \\
\text { Podsiadlo, 1981; Kozár } \text { et al.,1991; } \\
\text { present paper }\end{array}$ \\
\hline
\end{tabular}

Fam Asterolecaniidae

\begin{tabular}{|l|l|}
\hline Species & Validation source \\
\hline Pollinia pollini (A. Costa) & Alexandrakis, 1980a \\
\hline
\end{tabular}


Fam. Diaspididae

\begin{tabular}{|c|c|}
\hline Species & Validation source \\
\hline Abgrallaspis cyanophylli (Signoret) & Kozár et al.(1991); present paper \\
\hline Acanthomytilus intermittens (Hall) & Kozár et al., 1991 \\
\hline Adiscodiaspis ericicola (Marchal) & Present paper \\
\hline Aonidia mediterranea (Lindinger) & Present paper \\
\hline Aonidia lauri (Bouché) & Present paper \\
\hline Aonidiella aurantii (Maskell) & $\begin{array}{l}\text { Ayoutantis, 1940; DeBach \& Argyriou, } \\
\text { 1967; present paper }\end{array}$ \\
\hline Aonidiella yehudithae Ben-Dov & Ben-Dov, 2006; Jansen et al., 2010 \\
\hline Aspidiotus nerii Bouché & Koronéos, 1934; Ayoutantis, 1940 \\
\hline Aulacaspis rosae (Bouché) & Kozár et al., 1991 \\
\hline Carulaspis minima (Signoret) & Present paper \\
\hline Chrysomphalus dictyospermi (Morgan) & Ayoutantis, 1940 \\
\hline Diaspidiotus lenticularis (Lindinger) & Present paper \\
\hline Diaspidiotus osborni (Newell \& Cockerell) & Kozár et al. 1991 \\
\hline Duplachionaspis berlesii Leonardi & Present paper \\
\hline Duplachionaspis natalensis (Maskell) & Kozár et al., 1991 \\
\hline Dynaspidiotus britannicus (Newstead) & Koronéos, 1934 \\
\hline Dynaspidiotus greeni Balachowsky) & Kozár et al., 1991 \\
\hline Getulaspis bupleuri (Marchal) & Present paper \\
\hline Gonaspidiotus minimus (Leonardi) & Jansen et al., 2010; present paper \\
\hline Hemiberlesia lataniae (Signoret) & Rosen \& DeBach, 1979; Podsiadlo,1983 \\
\hline Koroneaspis aegilopos (Koroneos) & Present paper \\
\hline Lepidosaphes beckii Newman & Ayoutantis, 1940 \\
\hline Lepidosaphes conchiformis (Gmelin) & Kozár et al., 1991 \\
\hline Lepidosaphes flava (Signoret) & Present paper \\
\hline Lepidosaphes ulmi (Linnaeus) & Podsiadlo, 1983; present paper \\
\hline Leucaspis löwi Colvée & Kozár et al., 1991 \\
\hline Leucaspis pusilla Löw & Podsiadlo, 1983; Jansen et al., 2010 \\
\hline Leucaspis riccae Targioni Tozzetti & Lindinger, 1912; present paper \\
\hline Lineaspis striata (Newstead) & Panis, 1981; present paper \\
\hline Mercetaspis halli (Green) & Podsiadlo, 1983 \\
\hline Oceanaspidiotus spinosus (Comstock) & Present paper \\
\hline Odonaspis ruthae Kotinsky & Present paper \\
\hline Parlatoria oleae (Colvée) & Argyriou, 1967; present paper \\
\hline Parlatoria parlatoriae (Šulc) & Jansen et al., 2010 \\
\hline Parlatoria ziziphi (Lucas) & Ayoutantis, 1940 present paper; \\
\hline Pseudaulacaspis pentagona (Targioni Tozzetti) & Kozár et al., 1991; present paper \\
\hline Prodiaspis tamaricicola (Malenotti) & Present paper \\
\hline Rhizaspidiotus donacis (Leonardi) & Kozár et al., 1991 \\
\hline Unaspis euonymi (Comstock) & Present paper \\
\hline
\end{tabular}


(C.J.Hodgson). Its presence in the island was previously generally recorded by Argyriou (1984) and later confirmed by Hodgson \& Gounari (2006).

\section{Fam. Pseudococcidae}

*Chorizococcus rostellum Lobdell: Iraklion, 21-26.ix.2009, Sorghum halepense, unidentified Asteracea; Knossos, 25.ix.2009, Piptatherum miliaceum (F. Kozár).

*Coccidohystrix sp. n.: Knossos, 25.ix.2009, Rosmarinus sp. (F. Kozár).

*Heterococcus nudus Green: Sorghum halepense, Knossos, 25.9.2009 (F. Kozár).

Planococcus citri (Risso): Iraklion, 21-26.ix.2009, Parietaria sp., Cynodon dactylon, unidentified Asteracea (F. Kozár); collected also by pheromone traps in Iraklion.

Planococcus vovae (Nasonov): Chania, 23.iii.1997, Cupressus sp. (G. Pellizzari).

*Pseudococcus longispinus (Targioni Tozzetti): Chania, 8.iv.2010, Pittosporum sp. (G. Pellizzari).

Rhizoecus albidus Goux: Iraklion, 21-26.ix.2009, Cynodon dactylon (F. Kozár).

*Spilococcus halli (McKenzie \& Williams): Iraklion, 21-26.ix.2009, Cynodon; Knossos, 25 ix.2009, Piptatherum miliaceum (F Kozár). Previously reported for the Greece mainland by Kozár (1985) as Chorizococcus viktorinae.

Trionymus aberrans Goux: Knossos, 25.ix.2009, Piptatherum miliaceum (F. Kozár). Previously recorded in Iraklion by Kozár et al. (1991).

*Trionymus multivorus (Kiritchenko): Iraklion, 21-26.ix.2009, undetermined Asteracea (F. Kozár).

\section{Fam. Eriococcidae}

*Acanthococcus sp. n.: Knossos, 25.ix.2009, host plant unknown (F. Kozár).

* Acanthococcus desertus (Matesova): Knossos, 25.ix.2009, Piptatherum miliaceum (F. Kozár)

\section{Fam. Kermesidae}

*Kermes palestiniensis Balachowsky: Aradena, 35¹3’22”N 2403'42”S, 7.iv.2010; 04.vi.2011 Quercus coccifera (G. Pellizzari, F. Porcelli, S. Convertini).

Kermes vermilio Planchon: Aradena, 35¹3'22'N 2403'42”S, 04.vi.2011 Quercus coccifera ( S. Convertini).

\section{Fam. Coccidae}

*Ceroplastes floridensis Comstock; Iraklion, 35²0'16”N, 2508'06”'E, 10.iv.2010, Citrus sp., Laurus nobilis, Ficus microcarpa (G. Pellizzari, G. Seljak, F. Kozár).

Ceroplastes rusci (Linnaeus): Iraklion, 21-26.ix.2009, Pittosporum sp. (F. Kozár); Chania, 9.iv.2010, Ficus carica, Nerium oleander (G. Pellizzari); Aptera, 11.iv.2010, Nerium oleander (C.J. Hodgson). 
*Ceroplastes sinensis Del Guercio: Chania - MAICh, 9.iv.2010, Myrtus communis (G. Pellizzari).

Coccus hesperidum Linnaeus: Iraklion, 21-26.ix.2009, Citrus sp. (F. Kozár); Kissamu, 7.iv.2010, Ficus microcarpa; Iraklion 10.iv.2010, Ficus sp., Laurus nobilis (G. Pellizzari). This very common species was first generically reported for Crete by Ayoutantis (1940) and later recorded in Knossos on Iris craetensis by Kozár et al. (1991).

*Lecanopsis formicarum (Newstead): Iraklion, 23 .ix.2009, Koeleria sp. (F. Kozár).

Lichtensia viburni Signoret: Episkopi, 3.iv.2010, Olea europea (C.J. Hodgson).

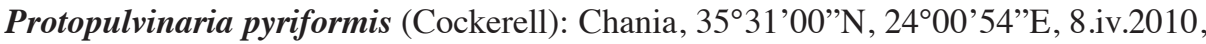

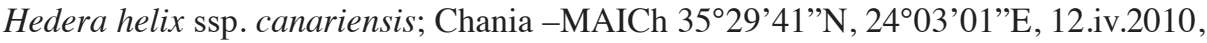
Hedera helix (G. Seljak).

Pulvinariella mesembryanthemi (Vallot): Chania, 8.viii.1975, Mesembryanthemum sp. (G. Pellizzari).

Sphaerolecanium prunastri Fonscolombe: Chania, 9.iv.2010, Ficus carica, (G. Pellizzari); Knossos, 35¹7'50”N , 2509’48”E, 10.iv.2010, Prunus dulcis (G. Seljak, F. Porcelli).

\section{Fam. Diaspididae}

Abgrallaspis cyanophylli (Signoret): Chania - MAICh $35^{\circ} 29^{\prime} 41^{\prime \prime} \mathrm{N}, 2^{\circ} 03^{\prime} 01^{\prime \prime} \mathrm{E}$, 6.iv.2010, Opuntia ficus-indica (G. Seljak, G. Pellizzari). Previously recorded in Iraklion, on Mesembryanthemum sp., by Kozár et al. (1991).

Acanthomytilus intermittens (Hall): Iraklion, 21-26.ix.2009, Cynodon dactylon, Piptatherum miliaceum (F. Kozár). Previously recorded in Iraklion and Knossos on Cynodon dactylon and Oryzopsis sp. by Kozár et al. (1991).

*Adiscodiaspis ericicola (Marchal): Elafonisi, 10.viii.2000, Erica manipuliflora, (F. Porcelli).

*Aonidia mediterranea (Lindinger): Chania, 23.iii.1997, Cupressus sp.,(G. Pellizzari); Iraklion, Cupressus sp., Thuja sp., 21-26.9.2009 (F. Kozár).

*Aonidia lauri (Bouché): Iraklion, 21-26.ix.2009, Laurus nobilis (F. Kozár).

Aonidiella aurantii (Maskell): Chania, 9.iv.2010, Euonymus sp. (G. Pellizzari)

Aspidiotus nerii Bouché: Aghia Sophia Cave - Topolia gorge, 01.viii.2000, Lavatera cretica \& Euphorbia sp. (woody srubs); Agria Gramvousa, 28.vii.2000, Thymelaea hirsuta; Bali, 29.vii.2000, Ceratonia siliqua (F. Porcelli). Iraklion, 21-26.ix.2009, Pittosporum tobira, Cercis sp., (F. Kozár). Previously recorded at Iraklion and Knossos, on Capparis spinosa, Nerium oleander, Pittosporum tobira, Sedum sp., Agave americana, Convolvolus sp., Eucayptus sp., Iris craetensis, Malva silvestris, Vitis sp. by Kozàr et al. (1991). *Carulaspis minima (Signoret): Iraklion, 21-26.ix.2009, Cupressus sp., Thuja sp. (F. Kozár).

*Diaspidiotus lenticularis (Lindinger): Potamida, 1.viii.2000, Olea europea, associated with and infected by Septobasidium sp. (F. Porcelli). 
*Duplachionaspis berlesii Leonardi: Chania-MAICh, 9.iv.2010, Asparagus acutifolius (G. Pellizzari).

*Getulaspis bupleuri (Marchal): Elafonisi, 15.vii.2000, Juniperus oxycedrus (F. Porcelli). Gonaspidiotus minimus (Leonardi in: Berlese \& Leonardi): Aradena, 35 13 '22'” N 2403'42”S, 04.vi.2011 Quercus coccifera (S. Convertini).

*Koroneaspis aegilopos (Koroneos): Aradena, 7.iv.2010, Quercus coccifera (G. Pellizzari).

*Lepidosaphes flava (Signoret): Potamida, 1.viii.2000, Olea europaea, associated with and infected by Septobasidium sp. (F. Porcelli).

Lepidosaphes ulmi (Linnaeus): Agyos Nicolaos, 8.viii.1975, Ceratonia siliqua (G. Pellizzari). Elafonisi, 15.vii.2000, Pistacia lentiscus (F. Porcelli).

*Leucaspis riccae Targioni Tozzetti: Aghia Sophia Cave - Topolia gorge, 1.viii.2000, Euphorbia sp. (woody shrub); Agria Gramvousa, 28.viii.2000, Euphorbia dendroides; Elafonisi, 01.viii.2000, Erica manipuliflora; Bali, 29.vii.2000, Olea europaea (F. Porcelli).

Leucaspis pusilla Löw: Chania, 9.iv.2010, Pinus halepensis (G. Pellizzari).

Lineaspis striata (Newstead): Agios Nikolaos, 10.viii.1975, Cupressus sempervirens (G. Pellizzari); Iraklion, 21-26.9.2009, Cupressus sp. (F. Kozár).

*Oceanaspidiotus spinosus (Comstock): Iraklion, 29.vii.2000, Opuntia sp. (F. Porcelli). *Odonaspis ruthae Kotinsky: Iraklion, 21-26.ix.2009, Sorghum halepensis, Cynodon dactylon (F. Kozár).

Parlatoria oleae Colvée: Iraklion, 21-26.ix.2009, Pyrus sp. (F. Kozár); Chania, 9.iv.2010, Prunus sp. (G. Pellizzari); Bali, 29.vii.2000, Pyrus pyraster (F. Porcelli).

Parlatoria ziziphi (Lucas): Potamida, 1.viii.2000, Citrus aurantium (F. Porcelli); Aptera, 12.iv.2010, Citrus lemon (C. J. Hodgson).

Pseudaulacaspis pentagona Targioni Tozzetti: Chania, 9.iv.2010; Chania- MAICh, by pheromone traps (F. Kozár); Aptera, 12.iv.2010, Morus sp. (C. J. Hodgson).

*Prodiaspis tamaricicola (Malenotti): Iraklion, 30.vii.2000, Elafonisi, 15.vii.2000, Tamarix gallica (F. Porcelli). Agios Nektarios, 35¹1'35”N, 24¹2'56”'E, 7.iv.2010, Tamarix gallica (G. Seljak).

*Unaspis euonymi (Comstock): Iraklion, 01.viii.2000, Euonymus japonicus (F. Porcelli).

According to the new collections and including the revised list from previous records, the total number of species recorded from the island is now 82 (table 1). Twenty-seven of them are new to the Crete fauna. Some new records refer to species widely distributed in the Mediterranean basin (i.e. Aonidia lauri, Carulaspis minima), whilst others are common introduced pests (i.e. Pseudococcus longispinus, Ceroplastes floridensis, Unaspis euonymi), so that their presence on the island was predictable. Significant records of interest are Kermes palestiniensis, previously only known to occur in Israel (Balachowsky, 1953) and Getulaspis bupleuri, previously known only from the Canary islands, North African countries (Morocco, Tunisia, Algeria, Lybia) and Saudi Arabia 
(Balachowsky, 1954; Matile Ferrero, 1984). The present list should be considered as a starting point for future biogeographic studies on the scale insects fauna of Crete.

\section{ACKNOWLEDGEMENTS}

The Authors wish to thank the University of Padova (Italy), for the scientific grant given to last Author for the cooperative work, the OTKA (Hungarian National Science Fund (Grant No. 75889) for financial support for this project and Chris Hodgson, for reviewing the manuscript and for allowing us to include his collecting data on Marchalina hellenica, Ceroplastes rusci, Lichtensia viburni, Parlatoria ziziphi, Pseudaulacaspis pentagona.

\section{REFERENCES}

AleXANDRAKIS V., 1980 - Essai d'appreciation des dégâts provoqués sur oranger en Crète par la presence d'Aonidiella aurantii (Mask.) (Hom. Diaspididae). Fruits 35: 555-560.

AleXandrakis V., 1980a - Données bio-ecologiques sur Pollinia pollini (Hom. Coccoidea, Asterolecaniidae) sur olivier en Crète. Annales de la Société Entomologique de France 16: 9-17.

ARGYRIOU L.C., 1967 - The scales of olive trees occurring in Greece and their entomophagous insects. Annales de l'Institut Phytopathologique Benaki (N.S.) 8: 66-73.

ARGYriou L.C., 1984 - Faunal analysis of some scale insects in Greece. Proceedings of the 10th International Symposium of Central European Entomofaunistics, Budapest, 15-20 August 1983: 364-367.

ARgYriou L.C., MichelaKis S., 1975 - Metaphycus lounsburyi Howard (Hymenoptera: Encyrtidae), parasite nouveau de Saissetia oleae Bern. en Crète, Grèce. Fruits 30: 251-254.

ARgYriou L.C., PAloukis S.S., 1976 - Some data on biology and parasitization of Sphaerolecanium prunastri Fonscolombe (Homoptera Coccidae) in Greece. Annales de l'Institut Phytopathologique Benaki (N.S.) 11: 230-240.

AyoutANTIS A., 1940 - Scale insects observed on citrus in the island of Crete. International Bulletin of Plant Protection 14: 2M-4M.

Balachowsky A.S., 1953 - Sur les Kermes Boitard (Hom. Coccoidea) des chênes du bassin oriental de la Méditerranée. Revue de Pathologie Végétale et d'Entomologie Agricole de France 32: 181-189.

Balachowsky A.S., 1954 - Les cochenilles Paléarctiques de la tribu des Diaspidini. Mémoires Scientifiques de l'Institut Pasteur, Paris, 450 pp.

Ben-Dov Y., 2006 - Taxonomy of Aonidiella yehudithae sp. nov., Lindingaspis misrae (Laing) comb. nov. with a key to species of Aonidiella Berlese \& Leonardi (Hemiptera: Coccoidea: Diaspididae). Zootaxa 1190: 51-57.

BEN-Dov Y., MilLER, D.R. GiBSON G.A.P., 2010 - ScaleNet: a database of the scale insects of the world. Available from http://www.sel.barc.usda.gov/scalenet/scalenet.htm (accessed June 2011).

Couch J.N., 1938 - The genus Septobasidium. The University of North Carolina Press. 479 pp.

Hodgson C.J., GounaRI S., 2006 - Morphology of Marchalina hellenica (Gennadius) (Hemiptera: Coccoidea: Marchalinidae) from Greece, with a discussion on the identity of $M$. caucasica Hadzibeyli from the Caucasus. Zootaxa 1196: 1-32. 
Hoy J.M., 1963 - A catalogue of the Eriococcidae (Homoptera: Coccoidea) of the world. New Zealand Department of Scientific and Industrial Research Bulletin 150: 1-260.

Koroneos J., 1934 - Les Coccidae de la Grèce surtout du Pélion (Thessalie). I. Diaspinae. Athens, $95 \mathrm{pp}$.

KozÁr F., 1985 - New data to the knowledge of scale-insects of Bulgaria, Greece, and Rumania (Homoptera: Coccoidea). Acta Phytopathologica Academiae Scientiarum Hungaricae 20: 201-205.

KozÁr F., Paloukis S., Papadopoulos N., 1991 - New scale insects (Homoptera: Coccoidea) in the Greek entomofauna. Entomologia Hellenica 9: 63-68.

KoZÁR F., NAGY B., 1998 - New data to the distribution of some Palaearctic scale insects (Homoptera: Coccoidea). Folia Entomologica Hungarica 59: 53-56.

JANSEN G.M., BEN-Dov Y., KAYDAN B., 2010 - New records of scale insects from Crete Island, Greece (Hem., Coccoidea). Bulletin Société entomologique de France 115: 483-484.

Matile-Ferrero D., 1984 - Insects of Saudi Arabia Homoptera: Subordo Coccoidea. Fauna of Saudi Arabia 6: 219-228.

MENDEL Z., 1998 - Biogeography of Matsucoccus josephi (Homoptera: Matsucoccidae) as related to host resistance in Pinus brutia and Pinus halepensis. Canadian Journal of Forest Research 28: 323-330.

Milonas P.G., KozÁR, F., Kontodimas D.C., 2008 - List of scale insects of Greece. 143-147 In: Branco, M., Franco, J.C. \& Hodgson, C.J. (Editors), Proceedings of the XI International Symposium on Scale Insect Studies, Oeiras, Portugal, 24-27 September 2007. ISA Press, Lisbon, Portugal, 322 pp.

PANIS A., 1981 - Les cochenilles circum-Méditerranéennes des arbres d'alignement et brise-vent (Homoptera, Coccoidea). Sixièmes Journées Phytiatrie and Phytopharmacie Circum-Mediterranéennes: 1-11.

Podsiadlo E., 1983 - Notes on scale insects (Homoptera, Coccoidea) found in Crete and their parasites. Fragmenta Faunistica 27: 271-277.

Rosen, D., DeBACH, P. 1979 - In: Species of Aphytis of the world (Hymenoptera: Aphelinidae). Series Entomologica: vol. 17) Dr. W. Junk, The Hague, Boston, London, 801 pp.

WiLLIAMS D.J., 1993 - A new species of mealybug from Greece, the first from Europe belonging to the ant-attended genus Eumyrmococcus Silvestri (Hemiptera: Coccoidea: Pseudococcidae). Entomologist's Gazette 44: 216-220.

Williams D.J., Moghaddam M., 2000 (1999) - Mealybug species of the genus Planococcus Ferris in Iran (Homoptera: Coccoidea: Pseudococcidae) with a discussion of Planococcus vovae (Nasonov). Journal of Entomological Society of Iran 18: 32-43.

GiUsepPina PellizZari - Università di Padova, Dipartimento Agronomia Ambientale e Produzioni Vegetali, Viale dell’Università 16, 35020 Legnaro, Italy. E-mail: giuseppina.pellizzari@ unipd.it

FranCESCo PorCelli - Università di Bari Aldo Moro, DiBCA sez. Entomologia e Zoologia, via Amendola 165A, 70126 Bari, Italy.

GabriJel SelJAK - Agriculture and Forestry Service Nova Gorica, Pri hastu 18, SI-5000 Nova Gorica, Slovenia.

FEREnC KoZÁR - Hungarian Academy of Sciences, Plant Protection Institute, P.O. Box 102, Budapest, H 1525, Hungary.

Accepted 15 September 2011 